\title{
Conductivity and superlattice ordering in an icosahedral Al-Pd-Mn phase
}

\author{
M. J. Capitan, ${ }^{1}$ J. Alvarez, ${ }^{2}$ J. J. Prejean, ${ }^{3}$ and C. Berger ${ }^{4}$ \\ ${ }^{1}$ Instituto de Estructura de la Materia, CSIC, c/Serrano 119, 28006 Madrid, Spain \\ ${ }^{2}$ Fisica de la Materia Condensada, CIII, Universidad Autonoma de Madrid, 28049 Madrid, Spain \\ ${ }^{3}$ CRTBT-CNRS, Box 166, 38042 Grenoble-Cedex 6, France \\ ${ }^{4}$ LEPES-CNRS, Box 166, 38042 Grenoble-Cedex 9, France
}

(Received 5 March 2003; published 12 August 2003)

\begin{abstract}
"In situ" conductivity measurements on Al-Pd-Mn quasicrystals have been combined with synchrotron $\mathrm{x}$-ray-diffraction experiments for studying its behavior during phase transformations. In order to confidently reduce the possible contribution to the conductivity of defects and grain boundaries, single-crystal samples have been annealed in situ under UHV conditions. This combined experiment allows us to affirm unambiguously that the structural state of the $i$-Al-Pd-Mn single crystal influences the electrical conductivity.
\end{abstract}

DOI: 10.1103/PhysRevB.68.064203

PACS number(s): 61.44.Br, 61.10.Nz, 71.30.+h

\section{INTRODUCTION}

In any condensed-matter system the atomic structure determines the potential felt by an electron, and thus, it has a fundamental influence on the electronic properties of the system. The discovery of the existence of alloys with a structure containing fivefold axes ${ }^{1}$ and, in consequence, having a quasiperiodic structure, opened a lot of questions about their electronic properties. However, only the relatively recent accessibility to thermodynamically stable quasicrystal phases of very high structural quality $(\mathrm{Al}-\mathrm{Cu}-\mathrm{Fe}, \mathrm{Al}-\mathrm{Cu}-\mathrm{Ru}$, and $\mathrm{Al}-$ $\mathrm{Pd}-\mathrm{Mn}$ systems $)^{2-4}$ allowed to relate unequivocally the quasi-periodicity with the exhibition of anomalous electronic and magnetic properties. ${ }^{5-10}$ Considering that the quasicrystals are entirely made of metals, they have a puzzling low conductivity. Their resistivity is $10^{3}-10^{4}$ times higher than the constituting metals and close to the exhibited by highly doped semiconductors. Moreover, the sharp increase of the conductivity versus temperature with no sign of saturation is a non-well-explained feature up to the moment. No opening of structural gap, contrary to the case of semiconductors, can account for this behavior, and the extremely high structural perfection of the single grains a priori excludes a lowtemperature localization mechanism induced by defects. Thus, there are still a lot of open questions about the electronic properties of the quasicrystals.

One of the features that has limited the comprehension of their electronic properties is the observed dependence of the conductivity with the sample. Thus, the conductivity depends on the sample preparation method (melt-spun ribbons or single crystal), on the sample composition, and even in some cases on the annealing treatment of the sample. The nonsystematic dependence of the conductivity with the annealing has been, at our knowledge, unexplored up to the moment. However, it has been reported that the electric properties of the single crystal sample can change after annealing within the temperature range from $600{ }^{\circ} \mathrm{C}$ to $800^{\circ} \mathrm{C}$ (Refs. 11, 12) in a nonsystematic direction. But in this range of temperature the existence of superlattice ordering with respect to the $\mathrm{P}$ lattice has been described in $\mathrm{Al}-\mathrm{Cu}-\mathrm{Fe}, \mathrm{Al}-\mathrm{Cu}-\mathrm{Ru}$, and $\mathrm{Al}-$ Pd-Mn single crystals for a chemical composition close to the thermodynamically stable icosahedral phase. ${ }^{2,13-15}$ This coincidence raises the question if there is a direct relationship between the sample superordering and its conductivity behavior. The aim of the present work is to combine in situ conductivity measurements with synchrotron x-raydiffraction studies in Al-Pd-Mn single-grain samples. Such in situ studies could allow to reduce the grain boundaries contribution to the conductivity and to clarify the influence of the atomic ordering in the electronic properties. Al-Pd-Mn has been chosen due to the high degree of perfection of the superordering structure. ${ }^{16-19}$

\section{EXPERIMENT}

In our studies two thermodynamically stable single grains of $\mathrm{Al}-\mathrm{Pd}-\mathrm{Mn}$ of atomic composition close to $\mathrm{Al}_{70} \mathrm{Pd}_{21} \mathrm{Mn}_{8}$ (Ref. 20) were used, as measured by inductively coupled plasma emission spectrometry. The single-crystal samples were cut from the core of single-crystal ingots pulled out from the melt by the Czochralski method after previous homogenization during 3 days at $1073 \mathrm{~K}$. Further annealing/ cooling procedures were applied to eliminate any stress produced during the polishing process and to yield high-quality quasicrystal structure with no secondary phases detected with synchrotron x-ray diffraction. This absence of secondary phases was also confirmed by high-precision magnetization measurements, which indicates no magnetic phases present (detection limit $\leqslant 1$ ppm Mn momentum).

The synchrotron $\mathrm{x}$-ray-diffraction experiments were carried out at the anomalous scattering beam line (ID01) at the ESRF. $^{21}$ The x-ray beam from the undulator source was monochromatized with a sagittal focusing double crystal $\mathrm{Si}(111)$ monochromator with cryogenics cooling. Highenergy harmonics were removed with two rhodium coated total reflection mirrors. The photon energy used was 10.76 $\mathrm{keV}$. The sample holder was inside an UHV chamber specially designed for this experiment coupled to a six-circle diffractometer allowing in situ sample annealing and x-raydiffraction and conductivity measurements. The sample was kept in a reflection configuration and the chamber pressure 


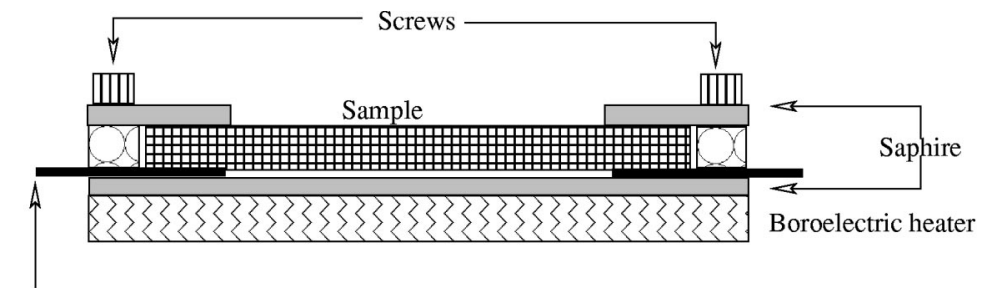

Tantalum

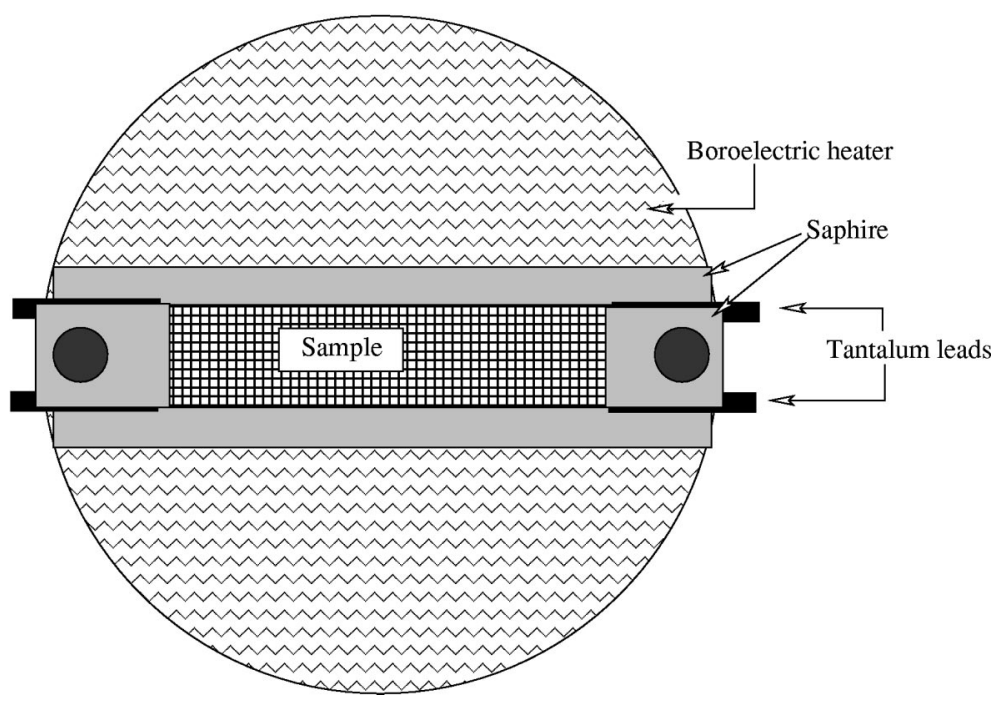

was kept under $10^{-8}$ mbar in the whole experimental temperature range. The outgoing beam was detected using a scintillator detector combined with an InSb (111) crystal analyzer to reduce the background signal.

The resistance $R$ of the samples was measured with the four-probe configuration. The in situ high-temperature relative resistivity $[\rho(T)]$ measurements were made in the Van der Paw configuration by pressing mechanically four electrical tantalum leads onto the sample (see Fig. 1). The absolute value of the resistivity was accurately measured elsewhere with small silver paste contacts. It is limited to a precision of $\Delta \rho / \rho=3 \%$ by the estimate on the sections and the distance between the voltage contacts. In order to account for possible pores in the material, the section $(s)$ is estimated from the sample weight $(m)$ according to $s=m / L d$ [ $L$ being the sample length and d the sample density equal to $5.1 \mathrm{~g} / \mathrm{cm}^{3}$ (Ref. 22)]. The parallelism of the low-temperature conductivity for all the samples ${ }^{23}$ was used as a test of accuracy of the obtained resistivity values. In the present experiment, due to the large sample size, the sample resistance was measured with a high-precision/low-noise ac bridge $(40 \mathrm{~Hz})$ from Barras instrument. At each measurement point the resistance bridge was balanced and the remaining deviation from equilibrium was measured with a Keithley nanovoltmeter. A precision of $10^{-3}$ was thus reached for resistance samples of less than $1 \mathrm{~m} \Omega$, measured $15 \mathrm{~m}$ apart in the $\mathrm{x}$-ray casemate. A thermocouple was pressed between a sapphire plate and the sample. Since we used a noncompensated feedthrough, the actual temperature was later reevaluated on the basis of previous high-temperature experiments on similar samples in a standard resistive furnace, with Pt-glued electrical contacts. $^{24,23}$
FIG. 1. Sketch of the heater sample holder and the tantalum leads used for the conductivity measurement. This dispositive is mounted inside a beryllium dome and continuously evacuated by a turbo pump. The vacuum was recorded during annealing, the pressure being lower than $10^{-8}$ bar during the whole experiment.

\section{RESULTS}

\section{A. The phase characterization}

The annealing of three Al-Pd-Mn single grains with an atomic composition close to $\mathrm{Al}_{70} \mathrm{Pd}_{21} \mathrm{Mn}_{8}$ reveals the existence of three different structures. Due to their structural complexity the $\mathrm{x}$-ray-diffraction characterization of these structures is presented before showing the combined study with the exhibited conductivity. In this way, it should be easier for the reader to know the phase at which authors refer later during the presentation of the in situ combined study.

Figure 2 presents the $\mathrm{x}$-ray-diffraction pattern in logarithmic scale obtained along a two-fold axis for the three different structures obtained. The diffraction pattern shown in the lower panel of Fig. 2 corresponds to the very well known quasicrystal icosahedral structure. ${ }^{1,15,25}$ Let us recall to the reader that the atomic structure of the icosahedral phases is described in a six-dimensional (6D) cubic lattice which decomposed into two three-dimensional (3D) spaces: the physical space $E_{\|}$and a complementary perpendicular space $E_{\perp}$. Thus, the 6D reciprocal space decomposes into two 3D reciprocal vectors $Q_{\|}$and $Q_{\perp}, Q_{\|}$being the experimental reciprocal momentum. It can be observed that all peaks can be assigned with the six indices following the scheme proposed by Cahn et al. ${ }^{26}$ In this case the derived $h / h^{\prime}, k / k^{\prime}, l / l^{\prime}$ indices have been used. The theoretical Bragg-peak position (vertical dashed lines in the figure) was obtained considering an icosahedral F-type structure with a lattice parameter two times the six-dimensional lattice parameter [in this case $a_{6 D}=6.462 \AA$ (Refs. 27, 15) has been used]. The peak relative intensities (given by the height of the relative vertical lines) can also be well reproduced with a very simple ap- 


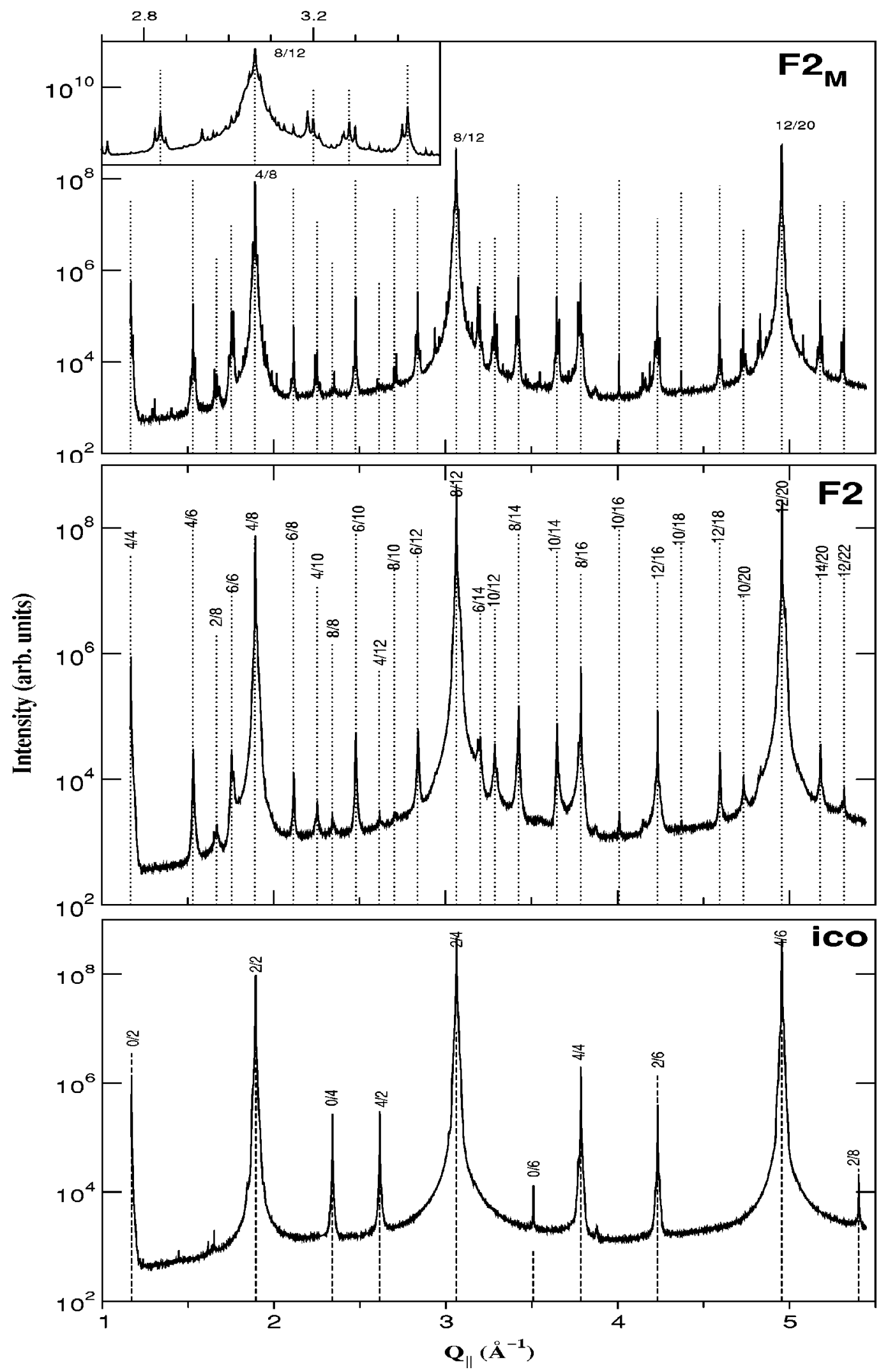

FIG. 2. X-ray-diffraction pattern along a twofold axis for the three obtained structures for the Al-Pd-Mn alloy close to $\mathrm{Al}_{70} \mathrm{Pd}_{21} \mathrm{Mn}_{8}$. In order to simplify the figure, only the $1 / 1^{\prime}$ indices are shown. Thus, the complete six-indices notation for these peaks should be $0 / 00 / 01 / 1^{\prime}$. The structures are shown in appearance order by increasing the temperature (from lower to upper panels) starting from a perfect icosahedral structure at room temperature. 


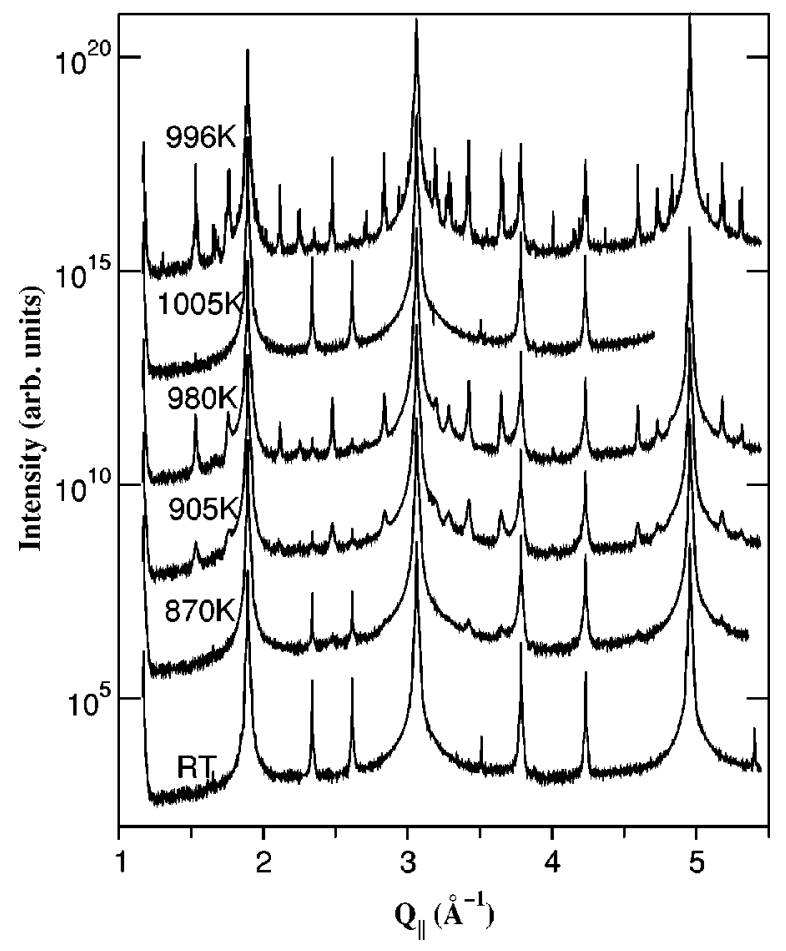

FIG. 3. Evolution of the x-ray-diffraction pattern along a twofold axis versus sample annealing temperature. The initial sample has an icosahedral structure at room temperature.

proximation by a model of spherical surfaces given by de Boissieu et al. ${ }^{25}$ in which the structure factor is modulated by the perpendicular space momentum $Q_{\perp}$ through a DebyeWaller factor $\left(F=F_{A S} e^{-B Q_{\perp}}, F_{A S}\right.$ being the structure factor for a rigid atomic surface).

Figure 3 shows the thermal evolution of an $\mathrm{Al}_{70} \mathrm{Pd}_{21} \mathrm{Mn}_{8}$ single-grain sample starting from the icosahedral phase at room temperature (RT). Depending very strongly on the sample composition, it has been observed that the annealing of some samples at low temperature (in the range 850-980 K) transforms this F-type icosahedral phase to another one. This new phase is characterized for a higher density of peaks, as can be appreciated by comparing its corresponding $\mathrm{x}$-ray diagram along a twofold axis shown in the middle panel of Fig. 2 with the one corresponding to the icosahedral F-type (lower panel of this figure). Although the highest Bragg peaks characteristic of the icosahedral phase do not change their intensities noticeably, one can observe the appearance of new peaks in between them and a deep decrease of intensity of some peaks of high $Q_{\perp}$ momentum characteristic of the icosahedral phase (i.e., 0/0 0/0 0/4, 0/0 0/0 4/2, $0 / 00 / 00 / 6$, and 0/0 0/0 2/8 Bragg peaks in Fig. 2). The resulting $\mathrm{x}$-ray pattern is totally compatible with a F2-type phase described in the literature. ${ }^{16-19}$ The structure transformation implied is given by a small breaking of F symmetry caused by superlattice ordering. The resulting structure has a P-type Bravais lattice which is related to the hightemperature F-type icosahedral phase (previously described) by $a_{P}=\tau a_{F}$, where $\tau$ and $a_{F}$ denotes the golden mean and the lattice parameter of the F-type icosahedral phase, respectively. In the present case the lattice parameter is $20.911 \AA$, which is in good agreement with values present in the literature. ${ }^{17}$ In the middle panel of Fig. 2 the peak position calculated for this lattice parameter has been pointed out (by means of vertical lines). A good agreement with the experimental data can be observed.

The thermal evolution of the Al-Pd-Mn single grain (see Fig. 3) reveals that the secondary Bragg peaks characteristic of this F2-type phase show an intensity broadening at the feet of the Bragg peak. Although the peak broadening decreases continuously with temperature (higher structural quality), these peaks keep broader than the Bragg peaks corresponding to the F-type icosahedral phase. Thus, two features describe the F2-phase quality: first, the Bragg-peak feet width and second, the disappearance of some Bragg peaks characteristic of the F-type phase associated to very high $Q_{\perp}$ values.

Above the F2-type temperature range, in a very narrow range, this structure evolves toward a highly dense Braggpeak structure (at $996 \mathrm{~K}$, in Fig. 3). This structural evolution depends on kinetic parameters. Starting from the F2-phase and increasing the temperature is very slow for the formation of this phase, on the contrary, it appears very rapidly following a very small temperature reduction coming from high temperature (F-type icosahedral structure formed above 1000 $\mathrm{K})$. This kinetic control makes it very difficult to define the temperature range of formation of this intermediate temperature phase. For the present sample it has been estimated within the temperature range of 980-1000 K. One very important point is that all the described heating-quenching processes have been shown as reproducible, in agreement with other literature data. ${ }^{18}$

This middle temperature phase has a x-ray pattern characterized by a higher density of Bragg peaks than the F2type structure, as can be observed in the upper panel of Fig. 2. The inset of this panel also shows clearly that these new peaks correspond to a splitting of the Bragg peaks characteristic of the low-temperature phase (F2 type). This structure shows a peak splitting with a reciprocal space distance of $0.0275 \pm 0.0005 \AA^{-1}$ along the threefold axis and 0.0130 $\pm 0.0005 \AA^{-1}$ along the twofold axis (upper and middle panels of Fig. 4, respectively). It seems that the peak splitting is worse defined along the twofold axis than along the threefold axis for the more intense Bragg peaks. In the middle and lower panels of Fig. 4, the peak splitting obtained along both directions for the same Bragg peak can be compared. However, this splitting along the twofold axis is more clear in the less intense F2-type Bragg peak (i.e., 0/0 0/0 6/12 and 0/0 $0 / 010 / 12$ in the middle panel of Fig. 4). The peak splitting obtained for the 0/0 0/0 8/12 F2 Bragg peak (which corresponds to a 20,32 Bragg peak of the F-type structure) has the same schema as that reported in the literature by Audier et al. ${ }^{19,18}$ in x-ray experiments. We obtained the same X-ray diagram along all the measured threefold cuts for this peak. That means that the cubic symmetry is preserved. Audier et al. ${ }^{19,18}$ show that this splitting corresponds to the formation of a sandwich structure of the F2-type phase with a well-defined period $\left(4 a+4 b\right.$, where $a=2 a_{F}$ and $b$ $\left.=2 \tau a_{F}\right)$, which has been observed in TEM investigations. ${ }^{19}$ Thus, those authors have assigned the origin of this phase 

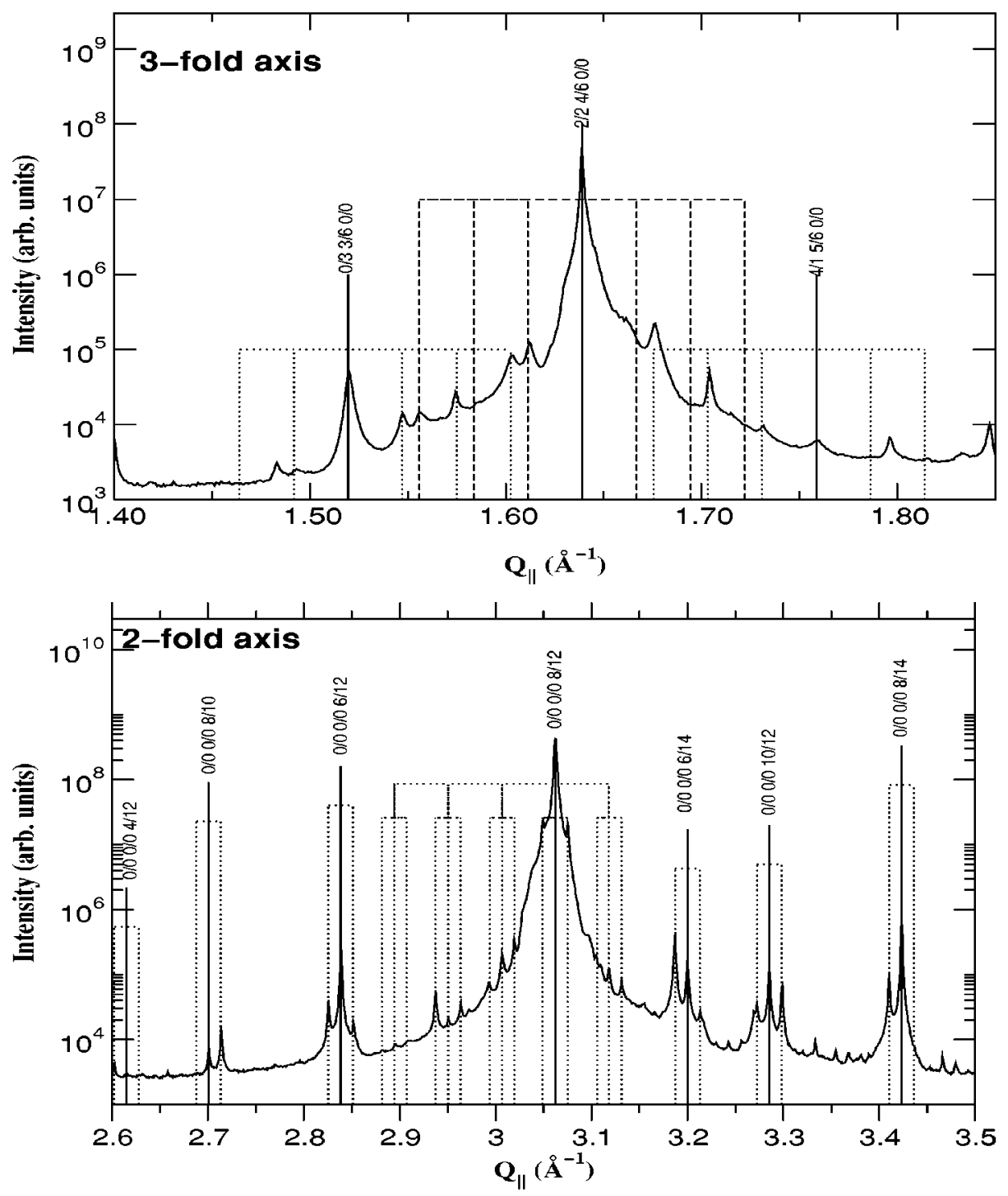

FIG. 4. X-ray-diffraction pattern along a twofold axis (medium panel) and the threefold axis (upper panel) for the middle temperature structure $\left(F 2_{M}\right)$. The most intense peak of the medium and upper panels corresponds, respectively, to the well-known $(20,32)$ and $(6,9)$ peaks in the $(N, M)$ notation of the F-type icosahedral phase (which corresponds to $0 / 0$ 0/0 8/12 and 2/2 4/6 0/0 respectively, in the F2 phase lattice). The lower panel shows the diffraction pattern of the $(20,32)$ icosahedral Bragg peak along a threefold direction.

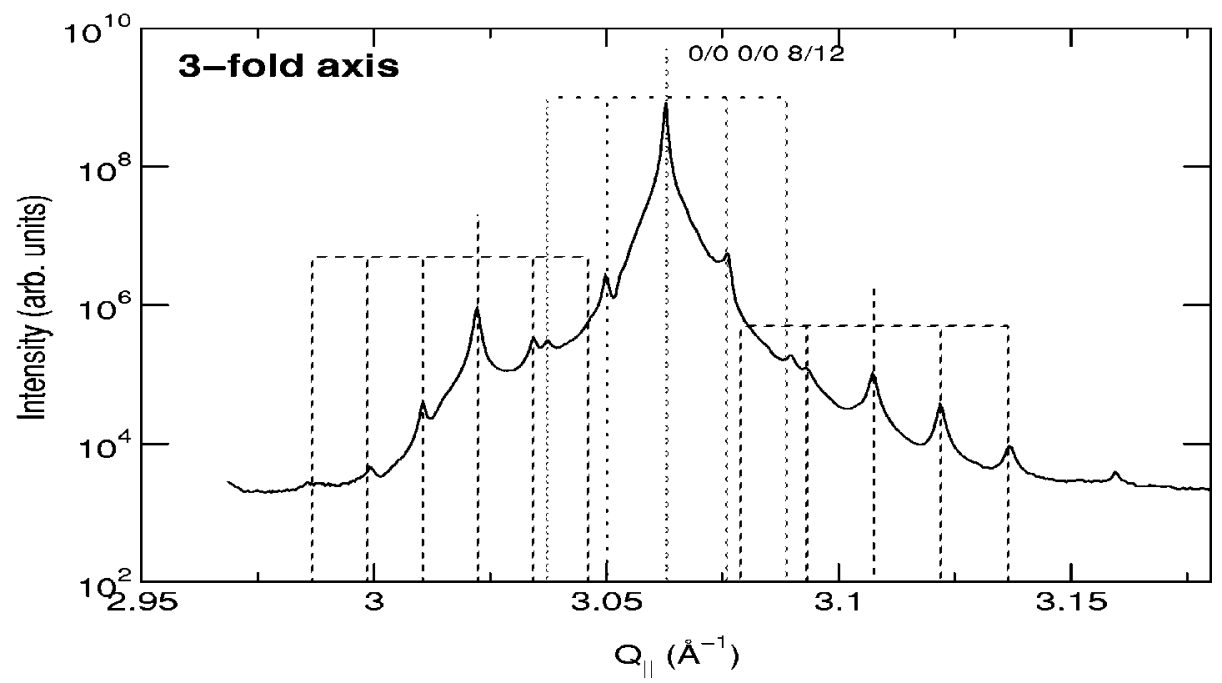

(which they call $\mathrm{F} 2{ }_{M}$ ) in terms of superlattice ordering of cubic symmetry in a superordered icosahedral phase F2. Its microstructure corresponds to multidomains with crystallographic orientation relationships between domains imparting an overall icosahedral symmetry.
From the electronic properties point of view, the correlation distance implied in the rearrangement of the F2 phase into the $\mathrm{F} 2{ }_{M}$ phase is too large with respect to the average distance for influencing the density of states for $d-s p$ coupled orbitals. ${ }^{12}$ The comparative studies of quasicrystals 


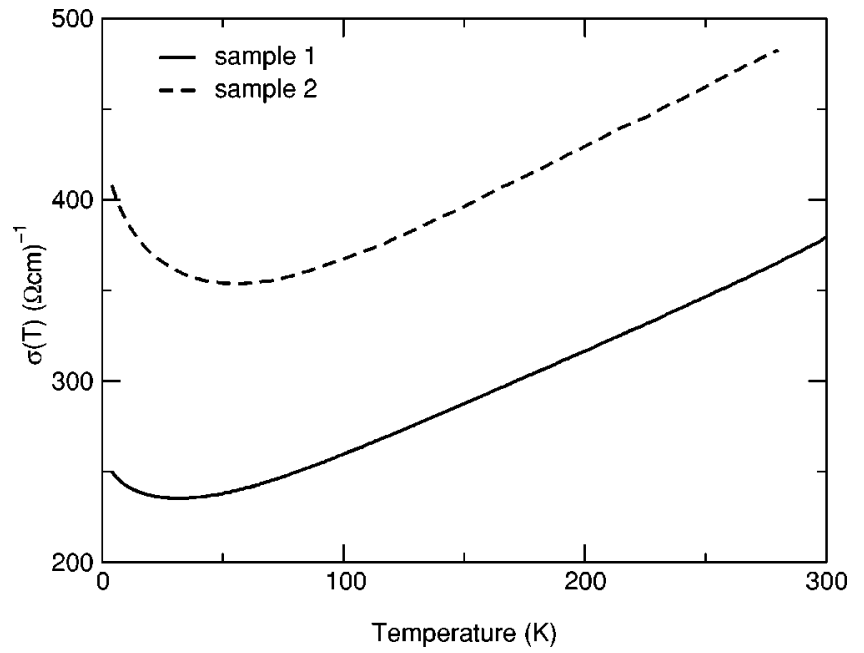

FIG. 5. Temperature dependence of the conductivity of two AlPd-Mn single-grain samples measured ex situ and previously to the diffraction experiment.

and approximant phases indicate that the main electronic features are determined by local order in the range of 15-30 approximately. ${ }^{12,24}$ Thus it seems reasonable to consider that the lower and intermediate temperature structures F2 type and $\mathrm{F} 2{ }_{M}$ type are going to influence equally the electronic properties of the alloys.

In summary, the F-type icosahedral phase is stable above $1000 \mathrm{~K}$. The low temperature stable phase is the $\mathrm{F} 2{ }_{M}$ phase while the $\mathrm{F} 2$ is a metastable intermediate state that appears when heating a previously quenched F-type phase. ${ }^{28}$

\section{B. Conductivity-phase dependence}

The absolute values of the conductivity measured "ex situ" for the studied samples in the low-temperature range of 4-300 K with a four probe configuration are shown in Fig. 5. These measurements were made previously to the simultaneous x-ray-diffraction and conductivity experiments. This figure shows the initial conductivity values for the two presented samples which were from two different ingots but with the same composition $\left(\mathrm{Al}_{70} \mathrm{Pd}_{21} \mathrm{Mn}_{8}\right)$. Both samples suffered different initial annealing treatment. The sample referred to as 1 corresponds to a rectangular plate-shape specimen which was annealed at $1073 \mathrm{~K}$ followed by a very slow cooling $(10 \mathrm{~K} / \mathrm{h})$ down to $773 \mathrm{~K}$. Sample 2, with the same shape, was previously annealed at $1073 \mathrm{~K}$ but followed by a rapid cooling $(150 \mathrm{~K} / \mathrm{min})$. Thus, in view of the phase temperature dependence shown in the preceding section, it was attempted to have different structural initial states for both samples. The corresponding initial conductivities shown in Fig. 5 show some characteristic features. The absolute conductivity (or its inverse resistivity value) at low temperature is of the same order of magnitude than the values reported in the literature for other high-quality $i$-Al-Pd-Mn phases. ${ }^{29,12}$ In the low-temperature range $(<50 \mathrm{~K})$ the conductivity presents a negative temperature dependence for both samples with an increasing slope at higher conductivity values. This dependence is predicted and observed for disordered alloys by the quantum interference effects and its origin is a weakly localized electron in space. Within a higher temperature range $(100-300 \mathrm{~K})$, the conductivities are parallel for both samples, a feature that can be used as test of accuracy of the measurement. ${ }^{23}$ This behavior has been observed in other quasicrystal alloys, and it implies that the conductivity can be decomposed on a constant independent term and a temperature-dependent one $\left[\sigma(T)=\sigma_{0}+\Delta \sigma(T)\right]$. The absolute value of the conductivity in quasicrystals is mainly governed by the temperature-independent term, which is a behavior opposite to that observed in a periodic metal.

The temperature dependence of the resistivity and the atomic structure were simultaneously recorded during the sample annealing. The sample phase was studied by means of synchrotron x-ray diffraction. The simultaneous resistivity data are shown in Fig. 6 by thick lines. The data are compared in this figure to their corresponding ex situ resistivity (thin lines) measured elsewhere previously (solid line) and after (dashed line) the simultaneous experiment. The upper panel corresponds to the sample noted as 1 and the lower panel to sample 2 . The different annealing treatment initially followed for both samples produced a different initial structural state in them as confirmed by x-ray diffraction and described in more detail hereafter. One of the advantages of such in situ studies is that they could allow to reduce the grain-boundary contribution to the conductivity clarifying the influence of the atomic ordering in the electronic properties.

The experimental procedure can be summarized as follows: Sample 1 is in equilibrium, $F 2_{M}$ state, then transformed to the F-type icosahedral phase, and quenched to room temperature to give the F-type phase. Sample 2 is in equilibrium, F-type state, then transformed to the $\mathrm{F} 2{ }_{M}$ phase and slowly cooled down to room temperature.

Sample 1 (upper panel of Fig. 6), which was previously slowly cooled down from $1073 \mathrm{~K}$, showed a diffraction pattern corresponding to a superordered quasicrystal structure (in this case, the $\mathrm{F} 2{ }_{M}$-type structure described in the preceding section). The structural state was monitored in the whole temperature range by $\mathrm{x}$-ray diffraction along the twofold axis of the sample. The temperature increase produces a transition from the super-ordered structure to the icosahedral structure above $1000 \mathrm{~K}$. The resistivity smoothly decreases by increasing the annealing temperature without any dramatic changes around the phase-transition temperatures (thick solid line). Once the sample transformed into the icosahedral phase, it was quenched, showing at RT the characteristic icosahedral (F-type) diffraction pattern. The resistivity measured in situ for the quenched sample at RT (holed cycles in Fig. 6) is lower than the initial value obtained for the superordered sample. In order to have the temperature dependence of the resistivity for the obtained icosahedral phase, this magnitude was monitored during a second annealing cycle. The resistivity of the icosahedral phase (thick dashed line) shows a shift down with respect to the superordered phase primarily measured, which starts at the end point of the first annealing cycle. This shift corresponds to a $12 \%$ increase in the temperature-independent term of the conductivity $\left(\sigma_{0}\right)$. During this second annealing cycle the sample changes from the 

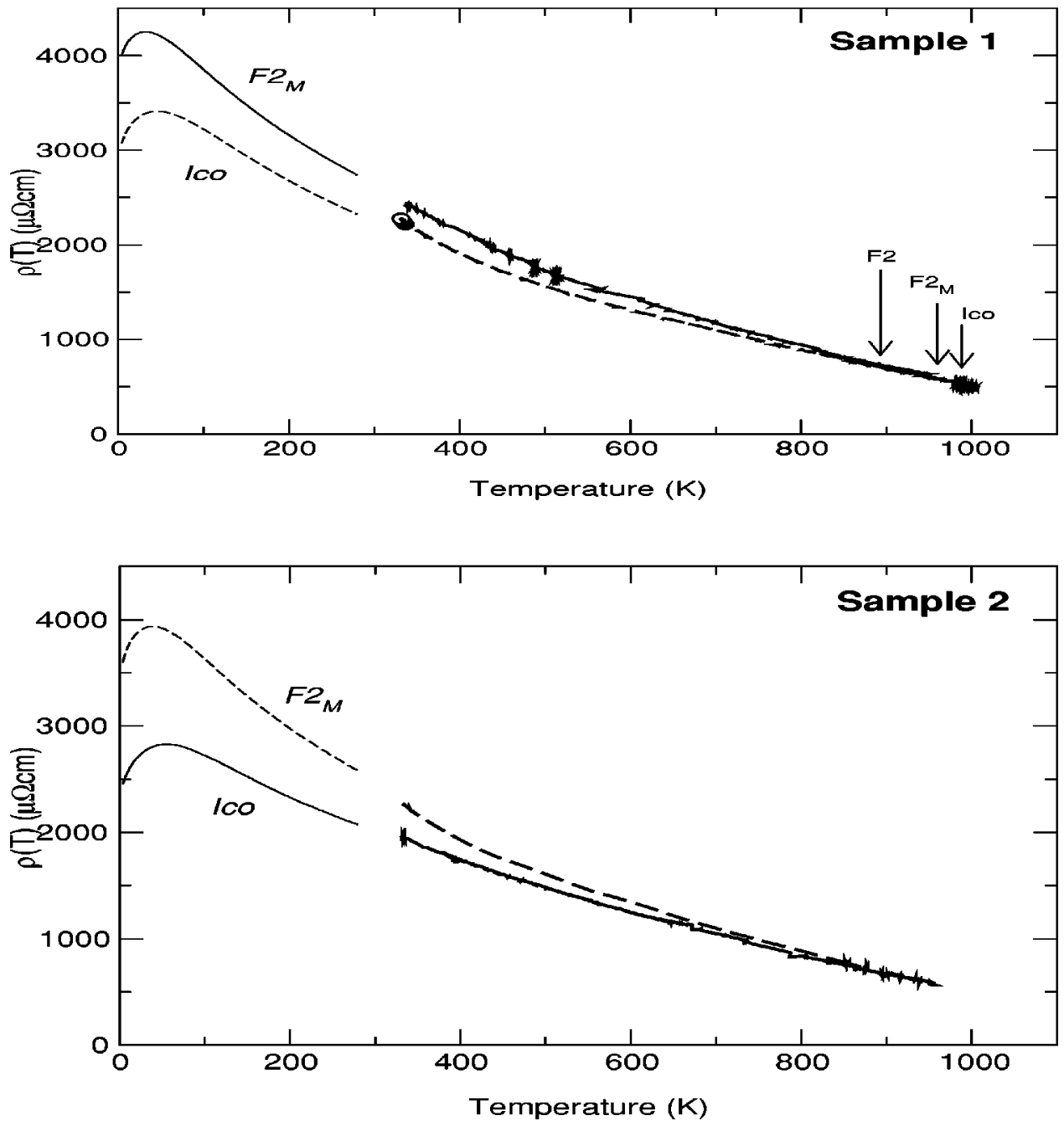

FIG. 6. Comparison of the temperature-dependent resistivity measured in situ at a high temperature (width lines), with the corresponding values obtained elsewhere (thin lines) using silver paste contacts in the lowtemperature range for two different samples as described in the text. The solid lines correspond to the initial annealing cycle (initial structural state) and the dashed line was obtained after the structural transformation (see text). icosahedral F-type phase to the superordered $\mathrm{F} 2$ and $\mathrm{F} 2{ }_{M}$, and again to $\mathrm{F}$ type by increasing the temperature (following the phase sequence shown in Fig. 3). The corresponding temperature transitions are indicated by arrows in Fig. 6. The resistivity also decreases by increasing the annealing temperature without any jumps around the phase-transition temperatures. The measured conductivity at the highest annealing temperature range are identical in both annealing cycles. It can be concluded that superordering increases the conductivity, the modification of the temperature independent term being its more evident sign. This phase dependence of the conductivity was confirmed with the conductivity measurement made ex situ to the initial and final sample (thin solid and dashed lines in Fig. 6, respectively).

In order to confirm this tendency and to discard any dependence of this behavior with the sample composition during annealing, other sample of the same composition (sample 2 ) has been studied, but with the phases in the inverse order. Sample 2 (lower panel of Fig. 6), which was quenched from $1073 \mathrm{~K}$, showed a diffraction pattern corresponding to the icosahedral phase, although a small mosaic block of F2-type structure was found in the opposite face to the measured one. The F-type sample resistivity decreased continuously by increasing temperature (thick solid line) with a tendency com- patible with the corresponding ex situ measurement (thin solid line). The phase structure of this sample was changed from icosahedral to $\mathrm{F} 2{ }_{M}$, passing through $\mathrm{F} 2$ in a temperature scheme equal to the one described in Fig. 3. Once the sample had a F2 ${ }_{M}$ structure, it was slowly cooled down. The cooling rate was slow enough to follow the resistivity during the cooling process (thick dashed line). In this case the resistivity shifts up with respect to the one measured for the initial icosahedral phase. This shift corresponds to a $9 \%$ decrease in the temperature-independent term of the conductivity $\left(\sigma_{0}\right)$.

Thus, the behavior of the conductivity versus annealing in both samples allows us to confirm several features. First, the presence of superordering in the sample increases the conductivity, manifested mainly as a modification of the temperature-independent term $\left(\sigma_{0}\right)$. Second, this shift is not very extended, being around $10 \%$ of its value. Third, the resistivity modification is not discontinuous and related with the temperature phase transition; in the icosahedral phase region they have the same value independent of their annealing history. The different shift sign obtained for the different annealing sequence allows to discard that the origin of the observed behavior is due to a sample composition change during annealing. 
In order to confirm this last point, a third experiment was made with another sample of the same ingot as the second one. The sample starts from the F-type icosahedral phase. The sample recovered the initial resistivity value within a $1 \%$ of error after a rapid quenching from the icosahedral annealing temperature. The conductivity does not change when no phase transition is produced.

The ex situ measurement made before and after the combined diffraction-conductivity experiment shows the same tendency to that described for the in situ experiment. The small differences between both experiments can be due to differences in the contacts or in the oxide layer of the samples.

\section{DISCUSSIONS}

The origin of the formation of the superordered structure from the icosahedral phase (referred to as F type) is not well defined up to the moment. Audier et al. ${ }^{19}$ suggest that it can be formed either from chemical or from topological ordering. This topological ordering can be produced either by a particular cut of the 6D hyperspace, whose serrated shape would be oscillating around the physical space, or due to a modification of the atomic surfaces in the $6 \mathrm{D}$ hyperspace. This latter possibility should imply that the atomic surface decoration changes in such way that they becomes much larger and less dense than in the icosahedral structure. Independent of the chemical or topological origin of the superordering structure, Ishimasa and Mori ${ }^{16}$ suggest that the existence of these phases implies that the so-called "ideal" and "perfect" quasicrystal (F-type icosahedral structure) still contain quasilattice imperfections or chemical disordering. Thus, both the superordered structures $\mathrm{F} 2$ and $\mathrm{F} 2{ }_{M}$ should correspond to a more perfect order than the icosahedral F-type structure. In the present experiment the formation of both superordered structure by sample annealing has been well characterized. These well-defined phases have been studied in large samples, the superorder being very extense.

On the other hand the relationship between the very low conductivity and the local atomic order is still not clear enough. However, Trambly de Laissaidiere and Mayou, ${ }^{30}$ in a theoretical work where they analyze the relationship between the tendency to electron localization and the local atomic order, show that these local environments can lead to resonance effects of the wave function in "clusters." Clusters in quasicrystals must be understood as a local atomic order, which is reproduced and is characteristic of the quasicrystal structure. The idea is the extension of the effect of one transition metal in a $s p$-metal matrix: in this case, there is a modification of the total $s p$ density of states due to the coupling with the $d$ orbitals. In this case the width of the peak produced in the density of states is proportional to the time that the electronic wave function spends in the vicinity of the transition-metal atom. The same effects of scattering electron is produced by clusters or group of clusters in quasicrystals. These clusters can lead to the formation of virtual bond states which are associated with narrow peaks in the density of states. Thus, Trambly de Laissaidiere and Mayou ${ }^{30}$ suggest that the virtual bond states are central to the elec- tronic structure of quasicrystals, producing a small modification of the total energy, so that the more perfect are such clusters (on a length scale of 10-30 $\AA$, the lower the conductivity. 31

By combining both the structural and the electronic studies it could be concluded that the superordered structures (F2 and $\mathrm{F} 2{ }_{M}$ ), which are more perfect, exhibit a lower conductivity than the F-type structure. This point can only be confirmed by a combined in situ experiment. In that way, the possible contribution to the conductivity of surface oxide coating, defects, and grain boundaries in single-crystal samples can be safely discarded. It has been observed that the temperature-independent term of the conductivity decreases by $10 \%$ when the quasicrystals transform from $\mathrm{F}$ type to a superordered structure. The possible contribution to the conductivity shift due to a compositional change in the samples during the annealing in UHV conditions can be excluded by our realization of experiments in both directions from icosahedral to superordered and vice versa in different samples, the conductivity being always $10 \%$ lower for the superordered phase. Moreover, the observed conductivity decrease is small, in agreement with the Hume-Rothery picture of quasicrystals, which implies that the tendency to the localization has a small effect on the total energy. ${ }^{30,12}$ Within the structure-conductivity dependence proposed in the literature ${ }^{31,30}$ it can be confirmed that the superordered structures have a higher local order than the icosahedral one. ${ }^{19,16}$ No evidence of differences between the two superordered phases $\mathrm{F} 2$ and $\mathrm{F} 2{ }_{M}$ can be deduced. It can be concluded that the structural differences between them are within a larger range than the length scale of $10-30 \AA$, which affects the conductivity. 31

Trambly de Laissaidiere and Mayou ${ }^{32}$ have shown the major role played by the $\mathrm{Mn}-\mathrm{Mn}$ interaction in the magnetic and conduction properties ${ }^{23}$ as well as in the emergence of quasiperiodicity. These authors have shown that the occurrence of localized magnetic moments is intrinsically linked to the quasicrystal stability. The present experiment shows unequivocally the direct relationship between the structural modification that produces the appearance of superorder and the conductivity. However, a possible explanation for the difference in conductivity could be related with the Mn atomic enviroment and position, which is known to play an important role in the transport properties of the $i$-Al-Pd-Mn. In that way the Mn atoms could have a different local order in the $\mathrm{F} 2_{M}$ phase resulting in a different conductivity. Although the Mn hypothesis matches with the present results, it cannot be assorted from them which atom ( $\mathrm{Al}, \mathrm{Pd}$, or $\mathrm{Mn})$ is at the origin of the observed phenomena.

The presented results have shown that the superorder mainly produces a modification in the $\sigma_{0}$ term of the conductivity. Taking into account that at high temperature the conductivity is mainly dominated by the $\Delta \sigma(T)$ term, the produced change in $\sigma_{0}$ can be neglected. A fit ${ }^{24}$ of the hightemperature part of the conductivity for the icosahedral structure and the $\mathrm{F} 2{ }_{M}$ phase, it is with $\sigma(T)=\alpha+\beta T^{3}$ gives an estimation of the $\sigma_{0}$ term. For the $\mathrm{F} 2{ }_{M}$ phase, it is 350.392 $(\Omega \mathrm{cm})^{-1}$, while for the icosahedral phase it is 370.13 $(\Omega \mathrm{cm})^{-1}$. In both cases the $\Delta \sigma(T)$ term at $900 \mathrm{~K}$ gives a 
contribution of $1159(\Omega \mathrm{cm})^{-1}$. In that way, the difference in $\sigma(T)$ between both structures at $900 \mathrm{~K}$ is around $1.3 \%$. That explains the lack of a jump in the resistivity around the phase-transition temperature.

\section{CONCLUSIONS}

In this paper we present the conductivity behavior of samples with very well-defined structures. A large effort has been devoted to the phase characterization and to determine the conditions for obtaining large samples with a single phase and a lower concentration of defects.

The combined x-ray-diffraction and conductivity studies show unequivocally a lower conductivity value when the superordered phase is formed. Independent of the chemical or topological origin of the superordering structure, several authors $^{16,19}$ agree that the existence of these phases implies that the so-called ideal and perfect quasicrystal (F-type icosa- hedral structure) still contains quasilattice imperfection or chemical disorder. So, this experiment confirms the structure-conductivity dependence proposed in the literature. ${ }^{31,30}$ These experiments show a $10 \%$ decrease in the conductivity when the superordered phase is formed with respect to the icosahedral phase. The low percentage of variation in the conductivity with order is in agreement with the Hume-Rothery picture of quasicrystals, which implies that the tendency to localization has a small effect on the total energy. ${ }^{30,12}$ It has also been pointed out that the origin of the superorder phase can be related to the local symmetry of the Mn atoms.

\section{ACKNOWLEDGMENTS}

The authors thank Y. Calvayrac and A. Kvick for providing the sample, the ESRF for the beamtime allocation, and J. J. de Miguel for careful reading.
${ }^{1}$ D. Shechtman, I. Blech, D. Gratias, and J.W. Cahn, Phys. Rev. Lett. 53, 1951 (1984).

${ }^{2}$ A.P. Tsai, A. Inoue, and T. Masumoto, J. Appl. Phys. 26, L1505 (1987).

${ }^{3}$ C.A. Guryan, A.I. Goldman, and P.W. Stephen, Phys. Rev. Lett. 62, 2409 (1989).

${ }^{4}$ Y. Calvayrac, A. Quivy, M. Bessiere, S. Lefebvre, M. CornierQuiquandon, and D. Gratias, J. Phys. (Paris) 51, 417 (1990).

${ }^{5}$ C. Berger and J.J. Prejean, Phys. Rev. Lett. 64, 1769 (1990).

${ }^{6}$ T. Klein, A. Gozlan, C. Berger, F. Cyrot-Lakmann, Y. Calvayrac, and A. Quivy, Europhys. Lett. 13, 129 (1990).

${ }^{7}$ B.D. Biggs, S.J. Poon, and N.R. Minirathnam, Phys. Rev. Lett. 65, 2700 (1990).

${ }^{8}$ B.D. Biggs, Y. Li, and S.J. Poon, Phys. Rev. B 43, 8747 (1991).

${ }^{9}$ V. Simonet, F. Hippert, M. Audier, and G. Trambly de Laissardiere, Phys. Rev. B 58, R8865 (1998).

${ }^{10}$ F. Hippert, V. Simonet, G. Trambly de Laissardiere, M. Audier, and Y. Calvayrac, J. Phys.: Condens. Matter 11, 10419 (1999).

${ }^{11}$ T. Klein, C. Berger, D. Mayou, and F. Cyrot-Lackmann, Phys. Rev. Lett. 66, 2907 (1991).

${ }^{12}$ C. Berger, in Lectures On Quasicrystals, edited by F. Hippert and D. Gratias, Les Editions de Physique (Les Ullis, France, 1994), p. 463.

${ }^{13}$ S. Ebalard and F. Spaepen, J. Mater. Res. 4, 39 (1989).

${ }^{14}$ A.P. Tsai, A. Inoue, Y. Yokohama, and T. Masumoto, Philos. Mag. Lett. 61, 9 (1990).

${ }^{15}$ M. Boudard, M. de Boussieu, C. Janot, J.M. Dubois, and C. Dong, Philos. Mag. Lett. 64, 197 (1991).

${ }^{16}$ T. Ishimasa and M. Mori, Philos. Mag. B 66, 513 (1992).

${ }^{17}$ T. Ishimasa, Philos. Mag. Lett. 71, 65 (1995).
${ }^{18}$ M. de Boissieu, M. Boudard, T. Ishimasa, E. Elkaim, J.P. Lauriat, A. Letoublon, M. Audier, M. Duneau, and A. Davroski, Philos. Mag. A 78, 305 (1998).

${ }^{19}$ M. Audier, M. Duneau, M. de Boissieu, M. Boudard, and A. Letoublon, Philos. Mag. A 79, 255 (1999).

${ }^{20}$ M.J. Capitan, Y. Calvayrac, A. Quivy, J.L. Joulaud, S. Lefebvre, and D. Gratias, Phys. Rev. B 60, 6398 (1999).

${ }^{21}$ M.J. Capitan, D. Thiaudiere, L. Goirand, R. Taffut, and S. Lequien, Physica B 283, 256 (2000).

${ }^{22}$ M. Boudard, Ph.D. thesis, Universite J. Fourier, Grenoble, France, 1993.

${ }^{23}$ J.J. Prejean, C. Berger, A. Sulpice, and Y. Calvayrac, Phys. Rev. B 65, 140203 (2002).

${ }^{24}$ D. Mayou, C. Berger, F. Cyrot-Lackmann, T. Klein, and P. Lanco, Phys. Rev. Lett. 70, 3915 (1993).

${ }^{25}$ M. de Boissieu, P. Stephens, M. Boudard, C. Janot, C. Chapman, and M. Audier, Phys. Rev. Lett. 72, 3538 (1994).

${ }^{26}$ J.W. Cahn, D. Schechtman, and D. Gratias, J. Mater. Res. 1, 13 (1986).

${ }^{27}$ M. J. Capitan, M. Bessiere, S. Lefebvre, Y. Calvayrac, A. Quivy, and D. Gratias, in Quasicrystals, edited by C. Janot and R. Mosseri (Avignon, France, 1995), p. 652.

${ }^{28}$ A. Letoublon, T. Ishimasa, M. de Boissieu, M. Boudard, B. Hennion, and M. Mori, Philos. Mag. Lett. 80, 205 (2000).

${ }^{29}$ S.J. Poon, Adv. Phys. 41, 303 (1992).

${ }^{30} \mathrm{G}$. Trambly de Laissaidiere and D. Mayou, Phys. Rev. B 55, 2890 (1997).

${ }^{31}$ D. Mayou, Phys. Rev. Lett. 85, 1290 (2000).

${ }^{32}$ G. Trambly de Laissaidiere and D. Mayou, Phys. Rev. Lett. 85, 3273 (2000). 